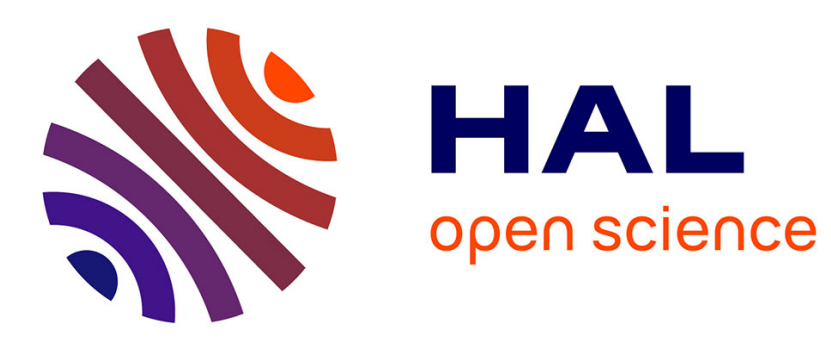

\title{
Low-Complexity Blind PAPR Reduction for OFDM Systems with Rotated Constellations
}

\author{
Tarak Arbi, Zi Ye, Benoit Geller
}

\section{To cite this version:}

Tarak Arbi, Zi Ye, Benoit Geller. Low-Complexity Blind PAPR Reduction for OFDM Systems with Rotated Constellations. IEEE Transactions on Broadcasting, 2021. hal-03426913

\section{HAL Id: hal-03426913 \\ https://hal.science/hal-03426913}

Submitted on 12 Nov 2021

HAL is a multi-disciplinary open access archive for the deposit and dissemination of scientific research documents, whether they are published or not. The documents may come from teaching and research institutions in France or abroad, or from public or private research centers.
L'archive ouverte pluridisciplinaire HAL, est destinée au dépôt et à la diffusion de documents scientifiques de niveau recherche, publiés ou non, émanant des établissements d'enseignement et de recherche français ou étrangers, des laboratoires publics ou privés. 


\title{
Low-Complexity Blind PAPR Reduction for OFDM Systems with Rotated Constellations
}

\author{
Tarak Arbi, Zi Ye and Benoit Geller
}

\begin{abstract}
The high Peak-to-Average Power Ratio (PAPR) of Orthogonal Frequency Division Multiplexing (OFDM) signals leads to a serious system performance degradation. To work around this issue, several algorithms have been proposed in the literature to reduce the PAPR, but, they often suffer from multiple limitations; in particular, the main issue with interleaving techniques is the spectral efficiency loss, as the transmission of a Side Information (SI) is generally required. In contrast to previous works, this paper proposes a blind interleaving technique for OFDM systems with signal space diversity. Indeed, with Rotated and Cyclically Q-Delayed (RCQD) constellations, the In-phase (I) and Quadrature (Q) components of constellations symbols are correlated, which allows the receiver to estimate the interleaver index without any SI. Moreover, to lower down the complexity burden at the receiver side, we first design a blind decoder based on the Minimum Mean Square Error (MMSE) criterion and we then propose a low complexity decoder for the Uniformly Projected RCQD (UP-RCQD) QAM, as this constellation has several interesting structural properties and achieves near optimum BER performance. Simulation results show that our proposal leads to a large PAPR reduction and to a near optimum BER performance that outperforms, over various channels, the solution currently used in DVB-T2. They also underline the good performance of the blind decoding performed with up to $98 \%$ of complexity reduction compared to the max-log Maximum Likelihood (ML) estimation.
\end{abstract}

Index Terms-OFDM, Peak-to-Average Power Ratio (PAPR), interleaving technique, embedded signalling, Rotated and Cyclically Q-Delayed (RCQD), Signal Space Diversity (SSD).

\section{INTRODUCTION}

To cope with the increasing need for high throughput communication systems over frequency selective channels, wireless communication standards often adopt the OFDM modulation because of several crucial advantages such as its low-complexity implementation and its high spectral efficiency. Usually, the various sub-carriers of the OFDM signal are modulated by classical QAM constellations, but recently the DVB-T2 standard included the possibility to use rotated constellation (RCQD) instead of ordinary QAMs [1]. Indeed, thanks to an inherent increased modulation diversity (Signal Space Diversity (SSD)), RCQD signals allow a better system performance over fading channels compared to conventional QAM signals [2]. To construct the two-dimensional (2D) RCQD signal, one first needs to rotate the conventional signals with a proper rotation angle and then to interleave the Q component, so as to ensure that the I and $\mathrm{Q}$ components of a given symbol experience independent

Tarak Arbi, Zi Ye and Benoit Geller are with Department U2IS of ENSTAParis, IP Paris, 828 Boulevard des Marechaux, 91120, Palaiseau, France (email: tarak.arbi,zi.ye,benoit.geller@ensta-paris.fr); fading channels [2]-[6]. At the receiver side, each component brings all the information carried by the original 2D symbol, which reduces the effect of fading.

The major drawback of this technique is the high soft demapping complexity; since the $\mathrm{I}$ and $\mathrm{Q}$ components of the same symbol experience different fading attenuations, low-complexity decorrelation-based methods, such as the MMSE demapper [7], lead to a poor system performance. In contrast, 2D demappers reach a near optimum performance; however the associated complexity is higher and leads to a considerable impact on the receiver design [8]. Recently, the authors in [9] proposed to rotate a QAM with $\mathrm{M}$ points by an angle $\theta=\arctan (1 / \sqrt{M})$ to design a low-complexity high-performance 2D sphere demapper for the so-called Uniformly Projected RCQD (UP-RCQD) constellation; this led to an electronic integration [10].

OFDM traditionally suffers from several limitations, such as the large PAPR introduced by the signal waveform that may lead to a considerable performance degradation and out-of-band radiation. Over the last decades, various techniques for reducing the PAPR have been proposed in the literature. They are commonly classified into three categories: signal distortion techniques, coding techniques and probabilistic techniques [11]. Signal distortion techniques introduce intentionally a distortion to the signal in order to reduce the PAPR. The clipping technique is the most basic method of this category, as it simply replaces the peaks of the OFDM signal by a predetermined threshold [12]. The key idea of coding techniques is to preselect an alphabet of codewords allowing a low PAPR [11]. Finally, several probabilistic techniques have been proposed in the literature; they are also known as distortionless techniques as they cause neither distortion to the signal, nor out-of-band radiation. The algorithms of this category can be classified into two subcategories: distortionless schemes with Side Information (SI) and distortionless techniques without SI. Indeed, several probabilistic techniques require the transmission of a side information such as the Tone Reservation (TR) scheme [13], [14], the conventional SeLected Mapping (SLM) [15]-[17], interleaving techniques [18] and the Partial Transmit Sequence (PTS) [19], [20]. On the other hand, various proposals have been made in the literature for which there is no need for any spectral spoilage, such as blind SLM at the cost of a rather high complexity [21] or Active Constellation Extension (ACE) better suited for small constellations [22]. The authors of [18] proposed an interleaving technique for PAPR reduction but 
this proposal requires a reliable transmission of the interleaver index; [23] proposed to embed the interleaver index into the OFDM pilot symbols but this may lead to a poor channel estimation at the receiver side [24] and it is not adapted to the case of slow fading channels as pointed out by [25].

To specifically reduce the PAPR of OFDM symbols when RCQD signals are used, the DVB-T2 standard recommends to only use a single PAPR reduction method (the TR method); indeed, contrarily to the case of conventional QAM signals, the ACE method and the TR method cannot be deployed together to further reduce the PAPR [1]. Therefore, [5], [6] propose a blind SLM technique with two RCQD constellations; the couple of rotation angles involved in this technique is obtained by jointly optimizing the theoretical system performance and the blind decoding performance. However, this method does not consider neither the high demapping complexity of RCQD signals, nor the high complexity introduced by the blind decoding. Therefore, in our proposal, an original low-complexity blind PAPR reduction technique is considered that takes advantage of the inherent structure of rotated constellations and for which a low-complexity MMSE-based blind decoder can be used; in addition, to lower down even more the complexity of our proposal, we also propose to interleave the $\mathrm{I}$ and $\mathrm{Q}$ components of rotated symbols by several possibilities of two-component interleavers and to use the rotation angles $\theta=\arctan (1 / \sqrt{M})$. The best symbol in terms of PAPR reduction is then transmitted. The high performance of the proposed max-log ML decoder allows the receiver to blindly identify the two-component interleaver used in the broadcast thanks to the inherent properties of rotated constellation. Compared to the ML estimation, our proposal reduces considerably the computational complexity while still achieving similar performance with no spectral spoilage.

The reminder of this paper is organized as follows. Section II presents our proposal and derives the ML estimator. Section III details the proposed low-complexity decoder and provides a complexity analysis of our proposal. After some simulation results in section IV, section $\mathrm{V}$ finally draws some conclusions.

\section{OVERVIEW OF THE PROPOSED BLIND PAPR REDUCTION TECHNIQUE WITH SSD} 1.

We now describe the communication system depicted in Fig.

\section{A. At the transmitter side}

Each message frame is first channel encoded and interleaved; then every block of $N\left(\log _{2}(M)\right)$ bits are converted into a series of $N$ complex-valued $M-Q A M$ symbols with Gray mapping:

$$
s(n)=s_{1}(n)+j s_{2}(n),
$$

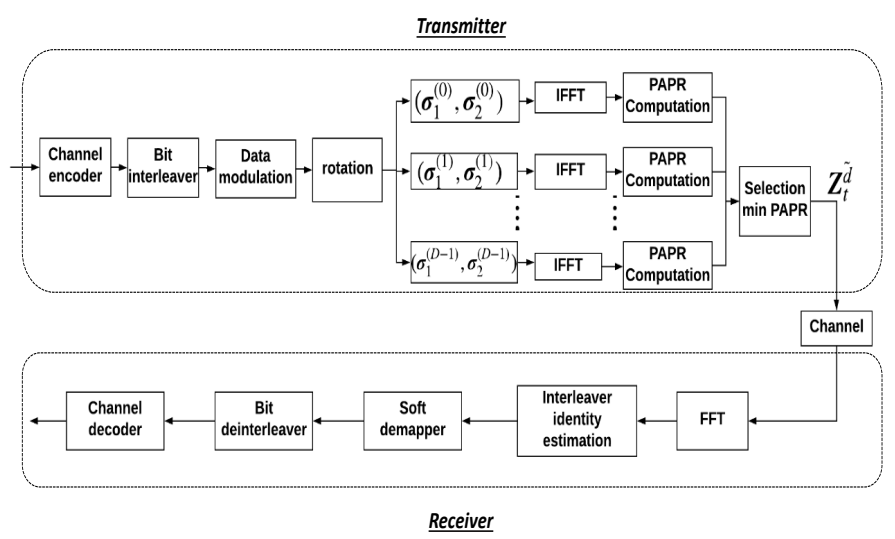

Fig. 1. System model.

where $s_{1}(n)$ and $s_{2}(n)$ are the I and Q components of $s(n)$ at time $n$; the two components can be expressed as:

$$
s_{i}(n)=2 p_{i}-\sqrt{M}+1,
$$

where $p_{i}$ takes integer values spanning from 0 to $\sqrt{M}-1$ and index $i$ belongs to $\{1,2\}$.

Then, the proposed transmitter rotates the QAM symbol as:

$$
x(n)=s(n) e^{j \theta}=x_{1}(n)+j x_{2}(n) .
$$

Traditionally, the second step of the RCQD modulation is to cyclically delay the $\mathrm{Q}$ components $x_{2}(n)$ so that the components $x_{1}(n)$ and $x_{2}(n)$ of the rotated symbol $x(n)$ experiment independent channels. In this paper, the transmitter possesses an alphabet of $D$ different two-component interleavers $\left\{\left(\sigma_{1}^{(d)}, \sigma_{2}^{(d)}\right), d=0,1, \cdots, D-1\right\}$; the transmitter generates in parallel $D$ OFDM symbols by applying each possible two-component (in-phase and quadrature) interleavers; one thus obtains, for every index $d$, the sequence:

$$
z_{f}^{(d)}(n)=x_{1}\left(\sigma_{1}^{(d)}(n)\right)+j x_{2}\left(\sigma_{2}^{(d)}(n)\right) .
$$

The transmitter then builds in parallel $D$ OFDM symbols:

$$
z_{t}^{(d)}(n)=\sum_{k=0}^{N-1} z_{f}^{(d)}(k) e^{\frac{2 \pi n k}{N}} .
$$

Before proceeding to any transmission, for each twocomponent interleaver $\left(\sigma_{1}^{(d)}, \sigma_{2}^{(d)}\right)$, the transmitter computes the corresponding $P A P R(d)$ as:

$$
P A P R(d)=\frac{\max _{n=0, \cdots, N-1}\left|z_{t}^{(d)}(n)\right|^{2}}{\left.\mathbb{E}\left(\mid \boldsymbol{Z}_{t}^{(d)}\right)\right|^{2}},
$$

where $\boldsymbol{Z}_{\boldsymbol{t}}^{(d)}=\left(z_{t}^{(d)}(0), z_{t}^{(d)}(1), \cdots, z_{t}^{(d)}(N-1)\right)^{T}$ and $\mathbb{E}$ denotes the expectation.

Thereafter, the transmitter selects index $\tilde{d}$ such that:

$$
\tilde{d}=\underset{d=0,1, \cdots, D-1}{\operatorname{argmin}} \operatorname{PAPR}(d),
$$


and only delivers the corresponding OFDM symbol at the channel input. Note finally that each two-component interleaver $\left(\sigma_{1}^{(d)}, \sigma_{2}^{(d)}\right)$ can be associated with a given bit interleaver at the transmitter side, in order to allow better PAPR reduction performance [18].

\section{$B$. Interleaver estimation at the receiver side}

The received signal after Fast Fourier Transform (FFT) can be expressed as:

$$
y(n)=H(n) z_{t}^{(\tilde{d})}(n)+w(n),
$$

where $H(n)$ is a Rayleigh random variable with unit variance and $w(n)=w_{1}(n)+j w_{2}(n)$, where $w_{1}(n)$ and $w_{2}(n)$ are independent zero-mean Gaussian random variables with known variance $N_{0}$. For a Rayleigh fading model with erasure events, $H(n)$ is equal to zero if an erasure event occurs, as defined in [1]. In addition, it is considered that the receiver has a perfect channel state information.

The receiver first has to deinterleave the received observations according to each possible two-component interleaver $\left(\sigma_{1}^{(d)}, \sigma_{2}^{(d)}\right)$, such that the deinterleaved received signals are:

$$
\begin{aligned}
r^{(d)}(n) & =r_{1}^{(d)}(n)+j r_{2}^{(d)}(n), \\
& =y_{1}\left(\left(\sigma_{1}^{(d)}\right)^{-1}(n)\right)+j y_{2}\left(\left(\sigma_{2}^{(d)}\right)^{-1}(n)\right) \\
& =h_{1}^{(d)}(n) x_{1}\left(\left(\sigma_{1}^{(d)}\right)^{-1}\left(\sigma_{1}^{(\tilde{d})}(n)\right)\right) \\
& +j h_{2}^{(d)}(n) x_{2}\left(\left(\sigma_{2}^{(d)}\right)^{-1}\left(\sigma_{2}^{(\tilde{d})}(n)\right)\right)+v^{(d)}(n),
\end{aligned}
$$

where $v^{(d)}(n)$ and $h_{i}^{(d)}, i \in\{1,2\}$, have the same statistical model characteristics as $w(n)$ and $H(n)$ respectively.

Two points should be noted; first, in order to have an increased signal space diversity, the two-component interleaver $\left(\sigma_{1}^{(d)}, \sigma_{2}^{(d)}\right)$ must guarantee that the I and $\mathrm{Q}$ of the rotated symbol $x(n)$ experience independent fading channels. This condition for instance, can be satisfied in practice by first estimating the coherence bandwidth of the channel and then by ensuring that, for all $k$, the bandwidth that separates the subcarrier of index $\sigma_{1}^{(d)}(k)$ and the one of index $\sigma_{2}^{(d)}(k)$ is larger than the coherence bandwidth. Second, in contrast to conventional QAM constellations, with a properly designed rotation angle, the $\mathrm{I}$ and $\mathrm{Q}$ component of $x(n)$ in (3) are correlated. This property inherently allows the receiver to estimate the index of the two-component interleaver used in the broadcast with no side information. Indeed, using the ML criterion, one can estimate, among the known alphabet of twocomponent interleavers, the one used in the broadcast such as:

$$
\begin{aligned}
\hat{d} & =\underset{d=0,1, \cdots, D-1}{\operatorname{argmax}} P\left(\boldsymbol{R}^{(d)}\right) \\
& =\underset{d=0,1, \cdots, D-1}{\operatorname{argmax}} \sum_{n=0}^{N-1} \log \left(P\left(r^{(d)}(n)\right)\right) \\
& =\underset{d=0,1, \cdots, D-1}{\operatorname{argmax}} \sum_{n=0}^{N-1} \log \left(\sum_{x \in \mathbb{X}} P\left(r^{(d)}(n) \mid x\right)\right),
\end{aligned}
$$

where $\boldsymbol{R}^{(d)}=\left(r^{(d)}(0), r^{(d)}(1), \cdots, r^{(d)}(N-1)\right)^{T}$ and $\mathbb{X}$ is the set of possible rotated symbols. Moreover, from (9) the probability $P\left(r^{(d)}(n) \mid x\right)$ can be developed as:

$$
P\left(r^{(d)}(n) \mid x\right)=\frac{1}{2 \pi N_{0}} e^{\frac{-\sum_{m=1}^{2}\left|r_{i}^{(d)}(n)-h_{i}^{(d)}(n) x_{i}\right|^{2}}{2 N_{0}}} .
$$

Using the max-log approximation in (11), (10) can then be simply evaluated with Euclidean distances as:

$$
\begin{aligned}
\hat{d} & =\underset{d=0,1, \cdots, D-1}{\operatorname{argmax}} \sum_{n=0}^{N-1} \max _{x \in \mathbb{X}}\left\{\log \left(P\left(r^{(d)}(n) \mid x\right)\right)\right\} \\
& =\underset{d=0,1, \cdots, D-1}{\operatorname{argmin}} \sum_{n=0}^{N-1} \min _{x \in \mathbb{X}}\left\{\sum_{m=1}^{2}\left|r_{i}^{(d)}(n)-h_{i}^{(d)}(n) x_{i}\right|^{2}\right\} .
\end{aligned}
$$

After having estimated the interleaver index $\hat{d}$, the receiver computes the Log-Likelihood Ratio (LLR) of the mapped bits. Finally, the deinterleaved LLR values are fed to the channel decoder in order to estimate the information bits $\hat{b}$.

\section{DETAILED PROPOSAL AND COMPLEXITY CONSIDERATIONS}

We now successively design a low-complexity MMSEbased decoder, enhance the analysis for the case of the Uniformly Projected RCQD QAM and analyze the complexity of the method proposed in section II.

\section{A. Low complexity MMSE-based decoder}

The deinterleaved received observations $r_{1}^{(d)}(n)$ and $r_{2}^{(d)}(n)$ in (9) can be rewritten as:

$$
\begin{aligned}
\left(\begin{array}{ll}
r_{1}^{(d)}(n) \\
r_{2}^{(d)}(n)
\end{array}\right)= & \left(\begin{array}{cc}
h_{1}^{(d)}(n) & 0 \\
0 & h_{2}^{(d)}(n)
\end{array}\right) \times \\
& \left(\begin{array}{l}
x_{1}\left(\left(\sigma_{1}^{(d)}\right)^{-1}\left(\sigma_{1}^{(\tilde{d})}(n)\right)\right) \\
x_{2}\left(\left(\sigma_{2}^{(d)}\right)^{-1}\left(\sigma_{2}^{(\tilde{d})}(n)\right)\right)
\end{array}\right)+\left(\begin{array}{l}
v_{1}^{(d)}(n) \\
v_{2}^{(d)}(n)
\end{array}\right) .
\end{aligned}
$$

For any interleaver index $d=\tilde{d},(13)$ can be developed as:

$$
\left(\begin{array}{l}
r_{1}^{(d)}(n) \\
r_{2}^{(d)}(n)
\end{array}\right)=\mathbf{A}^{(d)}(n) \times\left(\begin{array}{l}
s_{1}(n) \\
s_{2}(n)
\end{array}\right)+\left(\begin{array}{l}
v_{1}^{(d)}(n) \\
v_{2}^{(d)}(n)
\end{array}\right),
$$

where:

$$
\mathbf{A}^{(d)}(n)=\left(\begin{array}{cc}
h_{1}^{(d)}(n) & 0 \\
0 & h_{2}^{(d)}(n)
\end{array}\right)\left(\begin{array}{cc}
\cos (\theta) & -\sin (\theta) \\
\sin (\theta) & \cos (\theta)
\end{array}\right) .
$$

With an MMSE decorrelator, we then obtain:

$$
s^{M M S E}(n)=\left(\mathbf{A}^{(d)}(n)^{T} \mathbf{A}^{(d)}(n)+N_{0} \mathbf{I}\right)^{-1} \mathbf{A}^{(d)}(n)^{T}\left(\begin{array}{l}
r_{1}^{(d)}(n) \\
r_{2}^{(d)}(n)
\end{array}\right),
$$

where $\mathbf{I}$ is the identity matrix of size 2 .

Thereafter, the receiver searches the closest constellation point 


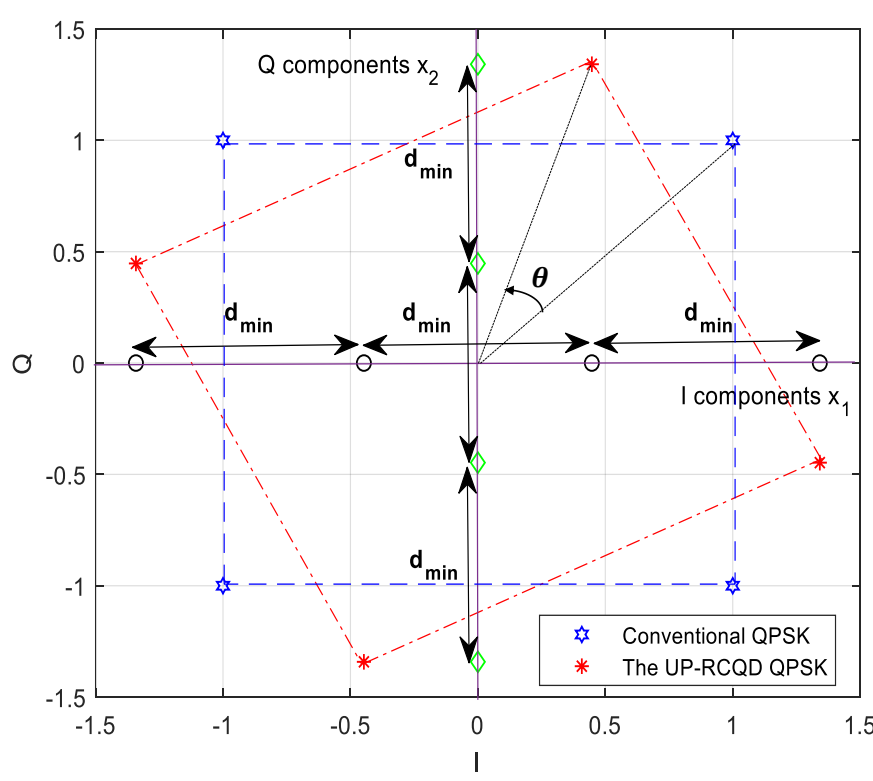

Fig. 2. The UP-RCQD QPSK signal.

to $s^{M M S E}(n)$ in terms of Euclidean distance and finds the corresponding rotated symbol $\hat{x}^{M M S E}(n)$.

Finally, the receiver decodes the interleaver index as:

$\hat{d}=\underset{d=0,1, \cdots, D-1}{\operatorname{argmin}} \sum_{n=0}^{N-1}\left\{\sum_{m=1}^{2}\left|r_{i}^{(d)}(n)-h_{i}^{(d)}(n) \hat{x}_{i}^{M M S E}(n)\right|^{2}\right\}$.

As shown in subsection III.C, this algorithm reduces considerably the complexity compared to to the max-log algorithm (see (12)) without any BER degradation for system operating at moderate to high SNR over fading channels; however, this method increases the noise level and its detection performance becomes poor when the $2 \times 2$ channel matrix is singular. Such is not the case for the Uniformely Projected RCQD decoder [9] and in the following subsection, we derive a low-complexity index interleaver decoder for the Uniformly Projected RCQD.

\section{B. Low complexity index estimation for the Uniformly Pro-} jected RCQD constellations

1) The Uniformly Projected RCQD transmitted signal: By selecting the rotation angle $\theta=\arctan (1 / \sqrt{M})$, the I (resp. Q) components of the rotated symbols are uniformly projected with a uniform minimum distance $d_{\min }=2 \sin \theta$ along the I (resp. Q) axes [9], as illustrated in Fig. 8 for the QPSK constellation. This allows to use a low-complexity demapper that takes advantage of working with integers; indeed, each constellation point $x$ corresponds to a unique 2D integer point $\left(T_{x, 1}, T_{x, 2}\right)$ defined as:

$$
\begin{aligned}
& T_{x, 1}=\frac{x_{1}}{2 \sin \theta}+\frac{(M-1)}{2}=\sqrt{M} p_{1}+\left(\sqrt{M}-1-p_{2}\right), \\
& T_{x, 2}=\frac{x_{2}}{2 \sin \theta}+\frac{(M-1)}{2}=\sqrt{M} p_{2}+p_{1} .
\end{aligned}
$$

It is worth noting that $T_{x, 1}$ and $T_{x, 2}$ take integer values in the set $\{0,1, \cdots, M-1\}$, and each $T_{x, 1}$ or $T_{x, 2}$ value identifies a single 2D rotated symbol $x$; indeed from the value of $T_{x, 1}$, the pair $\left(p_{x, 1}, p_{x, 2}\right)$ which uniquely defines the rotated symbol $x$ (see (2)), can be obtained as:

$$
\left\{\begin{array}{l}
p_{x, 1}=\left\lfloor\frac{T_{x, 1}}{\sqrt{M}\rfloor}\right. \\
p_{x, 2}=\sqrt{M}-1-\left(T_{x, 1}-\sqrt{M} p_{x, 1}\right),
\end{array}\right.
$$

where $\lfloor a\rfloor$ designates the integer part of $a$. Similarly, $\left(p_{x, 1}, p_{x, 2}\right)$ can also be obtained from the value of $T_{x, 2}$ as:

$$
\left\{\begin{array}{l}
p_{x, 2}=\left\lfloor\frac{T_{x, 2}}{\sqrt{M}}\right\rfloor \\
p_{x, 1}=T_{x, 2}-\sqrt{M} p_{x, 2}
\end{array}\right.
$$

Finally, it is worth mentioning that the mutual conversion between $\left(p_{x, 1}, p_{x, 2}\right)$ and $\left(T_{x, 1}, T_{x, 2}\right)$ can be implemented by a look-up table, as it is fixed for a given constellation.

2) Low-complexity interleaver index estimation: Non erased received components $y_{i}(i \in\{1,2\})$ in (8) can first be equalized as follows:

$$
\begin{aligned}
y_{e q, i}(n) & =\frac{y_{i}(n)}{2 H(n) \sin \theta}+\frac{(M-1)}{2} \\
& =T_{x, i}(n)+\frac{w_{i}}{2 H(n) \sin \theta},
\end{aligned}
$$

where $T_{x, i}$ are given by (18) - (19).

Similarly to (9), from (22) the deinterleaved equalized observations can then be expressed as:

$$
\begin{aligned}
r_{e q}^{(d)}(n) & =h_{1}^{(d)}(n) T_{x, 1}\left(\left(\sigma_{1}^{(d)}\right)^{-1}\left(\sigma_{1}^{(\tilde{d})}(n)\right)\right) \\
& +j h_{2}^{(d)}(n) T_{x, 2}\left(\left(\sigma_{2}^{(d)}\right)^{-1}\left(\sigma_{2}^{(\tilde{d})}(n)\right)\right)+\frac{v^{(d)}(n)}{2 H(n) \sin \theta}
\end{aligned}
$$

The distance term $\left|r_{i}^{(d)}(n)-h_{i}^{(d)} x_{i}\right|^{2}$ in (12), where $i$ can either be 1 or 2, can then be rewritten as (see (22)):

$$
\left|r_{i}^{(d)}(n)-h_{i}^{(d)}(n) x_{i}\right|^{2}=(2 \sin \theta)^{2}\left|h_{i}^{(d)}(n)\left(r_{e q, i}^{(d)}(n)-T_{x, i}\right)\right|^{2} .
$$

Using (24), the max-log based decoder (12) becomes:

$$
\hat{d}=\underset{d=0,1, \cdots, D-1}{\operatorname{argmin}} \sum_{n=0}^{N-1} \min _{T_{x} \in \mathbf{T}}\left\{\sum_{i=1}^{2}\left|h_{i}^{(d)}(n)\left(r_{e q, i}^{(d)}(n)-T_{x, i}\right)\right|^{2}\right\},
$$

where $\mathbf{T}$ is the set of possible points $T_{x}=\left(T_{x, 1}, T_{x, 2}\right)$.

Eq. (25) implies that one needs to find, for each $d$ and each $n$, the global optimum $T_{o p t}=\left(T_{o p t, 1}, T_{o p t, 2}\right)$ defined as:

$$
T_{\text {opt }}=\min _{T_{x} \in \mathbf{T}}\left\{\sum_{i=1}^{2}\left|h_{i}^{(d)}(n)\left(r_{\text {eq,i }}^{(d)}(n)-T_{x, i}\right)\right|^{2}\right\} .
$$

Among constellation points, one can easily find the local optimum $\operatorname{Loc}_{i}^{(d)}(n)=\left(\operatorname{Loc}_{i, 1}^{(d)}(n), \operatorname{Loc}_{i, 2}^{(d)}(n)\right)$ that minimizes 
$\left|h_{i}^{(d)}(n)\left(r_{e q, i}^{(d)}(n)-T_{x, i}\right)\right|^{2}$, with $i$ being either 1 or 2 , such that (see 22):

$$
\operatorname{Loc}_{i, i}^{(d)}(n)= \begin{cases}0, & \text { if } r_{e q, i}^{(d)}(n) \leq 0, \\ \operatorname{round}\left(r_{e q, i}^{(d)}(n)\right), & \text { if } 0 \leq r_{e q, i}^{(d)}(n)<(M-1), \\ M-1, & \text { if } r_{e q, i}^{(d)}(n) \geq(M-1) .\end{cases}
$$

For sake of complexity, instead of searching the global 2D optimum in (25) among all constellation points, we propose to limit the search to $1 \mathrm{D}$ regions centered around $r_{e q, i}^{(d)}(n)$ with radius $r_{a}$ where $i$ is in $\{1,2\}$. For $r_{a}>0$, these regions can be expressed as:

$$
\widetilde{\mathbf{T}}_{i}^{(d)}(n)= \begin{cases}\left\{0, \cdots, 2 r_{a}-1\right\}, & \text { if } r_{e q, i}^{(d)}(n)<r_{a}, \\ \left\{M-2 r_{a}, \cdots, M-1\right\}, & \text { if } r_{e q, i}^{(d)}(n) \geq M-r_{a}, \\ \left\{\left\lfloor r_{e q, i}^{(d)}(n)\right\rfloor-r_{a}+1 \cdots,\left\lfloor r_{e q, i}^{(d)}(n)\right\rfloor+r_{a}\right\}, \text { otherwise, }\end{cases}
$$

whereas for radius $r_{a}=0, \widetilde{\mathbf{T}}_{i}^{(d)}(n)$ contains only the local optimum $\operatorname{Loc}_{i}^{(d)}(n)$. Each point $T_{x}$ within $\widetilde{\mathbf{T}}_{i}^{(d)}(n)$ distinctively locates a pair $\left(p_{1}, p_{2}\right)$ and this pair distinctively determines a constellation point $\left(s_{1}, s_{2}\right)$. Thus, $\widetilde{\mathbf{T}}^{(d)}(n)=$ $\widetilde{\mathbf{T}}_{1}^{(d)}(n) \cup \widetilde{\mathbf{T}}_{2}^{(d)}(n)$ has at most $4 r_{a}$ constellation points. In particular, for $r_{a}=0, \widetilde{\mathbf{T}}^{(d)}(n)$ contains the two local optimum points $\operatorname{Loc}_{1}^{(d)}(n)$ and $\operatorname{Loc}_{2}^{(d)}(n)$ that may lead to the same constellation symbol, which is then the global optimum. To summarize, from (25) and (28), we choose the interleaver index as:

$$
\hat{d}=\underset{d=0,1, \cdots, D-1}{\operatorname{argmin}} \sum_{n=0}^{N-1} \min _{T_{x} \in \widetilde{\mathbf{T}}^{(d)}(n)}\left\{\sum_{i=1}^{2}\left|h_{i}^{(d)}(n)\left(r_{e q, i}^{(d)}(n)-T_{x, i}\right)\right|^{2}\right\},
$$

We now present the successive steps of the proposed algorithm:

1) For each received component, use (22) to transform the received components $y_{i}(n)$ into equalized versions $y_{e q, i}(n)$. If one component is erased, $y_{e q, i}(n)$ is set to zero and the corresponding 2D Euclidean distance terms are not considered in (29).

2) For each received component, locate the region $\widetilde{\mathbf{T}}_{i}^{(d)}(n)$ using (28) with radius $r_{a}$; then the distance from $r_{e q, i}^{(d)}(n)$ is computed for all points in $\widetilde{\mathbf{T}}_{i}^{(d)}(n)$.

3) For each $d$ and $n$, given the one dimensional (1D) distance terms already obtained in step 2, compute the missing ones in (29) so as to obtain the 2D distance terms from $\left(r_{e q, 1}^{(d)}(n), r_{e q, 2}^{(d)}(n)\right)$ for all points in $\widetilde{\mathbf{T}}^{(d)}(n)$. The minimum 2D distance in (29) is then selected.

4) Using the minimum distances obtained in step 3 , the interleaver index $\hat{d}$ can finally be obtained (see (29)).

\section{Complexity analysis}

The detailed analysis on the complexity of the proposed algorithms is evaluated in terms of Real Multiplications (RM), Real Comparisons (RC), Real Inversions (RI) and Real
Sums (RS), where RS can either designate a real addition or a real subtraction.

At the transmitter side, note that no multiplication by any phase sequence is required for our proposal; this reduces the complexity burden by $4 N(D-1) \mathrm{RMs}$ and $2 N(D-1) \mathrm{RSs}$ compared to the SLM algorithm in [5], [6].

Furthermore, at the receiver side, the total computational complexity of the proposed MMSE-based blind detector (see equations (15)-(17)) is $38 N D$ RMs, $15 D N$ RSs, $\left(2 \log _{2} M-2\right) D N \mathrm{RCs}$, and $D N$ RIs.

We now detail the complexity of the index interleaver decoder proposed for the Uniformly Project RCQD (i.e. (29)):

1) Equalizing one received component $y_{i}(n)$ into $y_{e q, i}(n)$ (see 22), requires $1 \mathrm{RM}, 1 \mathrm{RS}$ and $1 \mathrm{RI}$. Therefore, equalizing the received observations requires a total of $N$ RM, $2 N$ RSs and $2 N$ RIs (i.e. $2 \sin \theta$ is a known constant term and its multiplication by $H(n)$ is performed only once).

2) Locating the region $\widetilde{\mathbf{T}}_{i}^{(d)}(n)$ requires 2 RCs. Computing a 1D Euclidean distance requires $2 \mathrm{RM}$ and $1 \mathrm{RS}$. Therefore, this step takes a total of $4 N r_{a}$ (resp. $2 N$ ) RMs, $2 N r_{a}$ (resp. $\left.N\right) \mathrm{RSs}$ and $4 N \mathrm{RCs}$.

3) In step 3, for each $d$ and each $n$, at least $4 r_{a}$ (resp. $\left.2 r_{a}\right)$ 1D distance terms like $\left|h_{i}^{(d)}(n)\left(r_{e q, i}^{(d)}(n)-T_{x, i}\right)\right|^{2}$ have already been computed in step 2 ; thus computing the other $4 r_{a}$ (resp. $2 r_{a}$ ) 1D distance terms requires a total of $8 r_{a} N D$ (resp. $4 N D$ ) RMs and $4 N r_{a}$ (resp. $2 N$ ) RSs for $r_{a}>0$ (resp. $\left(r_{a}=0\right)$ ). Moreover, obtaining the 2D Euclidean distance terms requires an additional $4 D N r_{a}$ (resp $\left.2 D N\right)$ RS. Finally, selecting the minimum 2D Eucldiean distances terms requires $D N\left(4 r_{a}-1\right)$ (resp. $D N$ ) RCs.

4) In step 4, performing the sum of minimum distances requires $(N-1) D \mathrm{RS}$ and selecting $\hat{d}$ requires $D-1$ RCs.

Two points should be noted; first, the proposed PAPR reduction algorithm can be used with any RCQD signals with inherent increased modulation diversity. In particular, it can be used with the rotation angles currently used in DVB-T2 [1]. Second, for the proposed blind PAPR reduction technique, the choice of the rotation angles $\theta=\arctan (1 / \sqrt{M})$ lowers down the complexity twice; first, the soft demapper proposed in [9] can be deployed at the receiver end, as it reduces by more than $60 \%$ the complexity compared to the simplest solution currently used in DVB-T2. Moreover, both the first and the second step of the proposed estimation technique are already necessary for the demapping solution in [9]; therefore, the additional complexity related to the interleaver estimation is just $8 r_{a} N D$ (resp. $\left.4 N D\right)$ RMs, $\left(8 r_{a}+1\right) N D-D$ (resp. $N D) \mathrm{RSs}$ and $\left(4 r_{a}-1\right) N D+D-1$ (resp. $\left.(N+1) D-1\right)$ RCs for $r_{a}>0$ (resp. for $r_{a}=0$ ). 


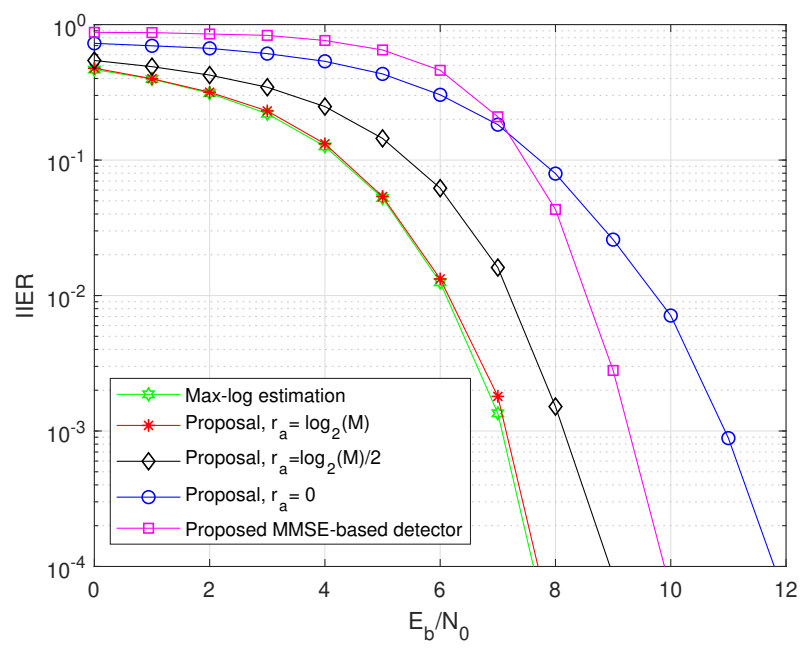

Fig. 3. IIER comparison between the full complexity max-log estimator, the proposed MMSE-based detector and the method for UP-RCQD constellations for several radii $r_{a}(64-\mathrm{QAM}, N=256$ and $D=16)$.

\section{Simulation Results}

Fig. 3 presents the Interleaver Index Error Rate (IIER) obtained with the max-log decoder and the proposed techniques (see (12), (17) and (25)) for several radii $r_{a}$ without any channel coding. It can be observed that there is roughly no difference between the proposed method with $r_{a}=\log _{2} M$ and the full complexity max-log decoder. Moreover, the performance deteriorates with the diminution of the radius; in particular, the proposed method with a radius $r_{a}=0$ respectively loses about $4 \mathrm{~dB}$ compared to the full-complexity max-log estimation and $2 \mathrm{~dB}$ compared to the MMSE based detector but it still operates at rather low SNR.

In the sequel, otherwise mentioned, we fix the following parameters : $N=256, D=16$ and 64-QAM. Fig. 4 compares the BER performance of the considered methods and the case where the receiver has an ideal SI of the interleaver index for several constellation sizes. In coherence with Fig. 3, for a system operating at moderate to high SNR regime, the considered methods lead to a low IIER and therefore to the same overall BER performance.

Moreover, Fig. 5 displays the BER curves, obtained for Rayleigh fading channels without and with $15 \%$ of erasure, for the DVB-T2 [1] system with a 64800-bit long LDPC code of rate $4 / 5$ with 25 -iterations min-sum decoding; the comparison is made for the angle of the UP-RCQD 64-QAM constellation used in our proposal, the angle currently used in DVB-T2, the rotation angle $\alpha_{1}$ obtained by minimizing an upper-bound on the BER [5] and the angle $\alpha_{2}=\arctan \left(\frac{1 \pm \sqrt{5}}{2}\right)$ known to be optimal for symbol error rate in the high SNR regime [2]. It can be observed that the UP-RCQD constellation achieves a near optimum performance over both channels; in particular, it outperforms the solution currently used in DVB-T2 [1] by about $1 \mathrm{~dB}$ over Rayleigh fading channels with $15 \%$ of erasure events. Similar results are obtained for other constellation

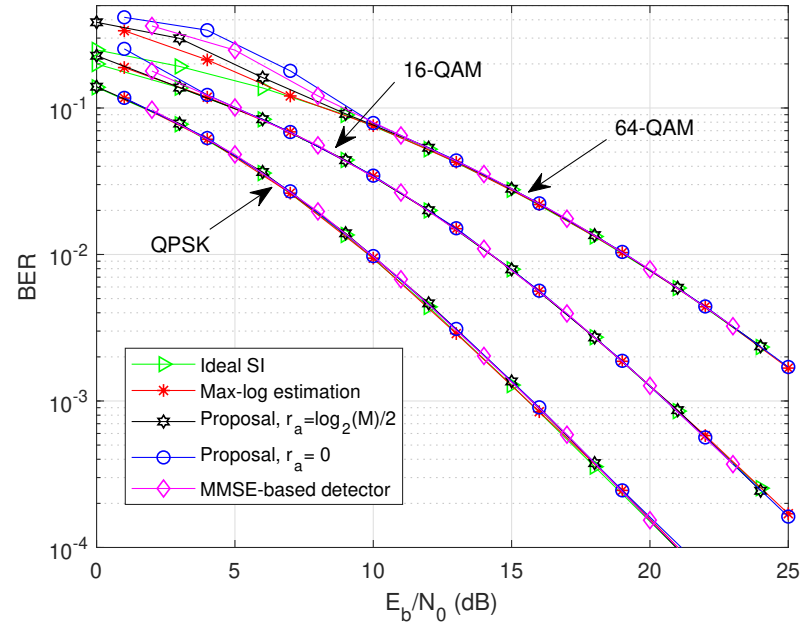

Fig. 4. BER comparison between the ideal RSI, the full complexity max$\log$ estimator, the proposed MMSE-based detector and the method for several radii $r_{a}$ over the Rayleigh channel without any erasure $(N=256, D=16)$.

sizes, coding rates and erasure rates (see [9]).

Considering both the transmitting and the receiving ends, Fig. 6 compares the total computational complexity, at both the transmitting and the receiving ends, between the max-log estimator, the MMSE-based detector and the proposed method for UP-RCQD constellations for several radii $r_{a}$ (see Table I); we assume that the cost of RC or RS is one, whereas, the cost of RM and RI are two and four respectively. First, we observe that the computational complexity of our proposal increases with the radius and that the complexity of the max$\log$ estimation is much higher than that of our section III proposals. For $M=256$, the MMSE-based detector achieves a $86 \%$ of complexity reduction compared to the max-log estimation. With the chosen system parameters, our proposal with $r_{a}=0$ achieves a $96 \%$ of complexity reduction compared to the MMSE-based detector; similarly, we obtain $84 \%$; of complexity reduction for the 4-QAM. In addition, it is worth mentioning that with the considered parameters, the system operates at $E_{b} / N_{0}=13 \mathrm{~dB}$ (see Fig. 5); therefore, the proposed decoder can work with a radius $r_{a}=0$ as it leads to a sufficiently low IIER (see Fig. 3).

Moreover, Fig. 7 presents for $N=1024$ the Complementary Cumulative Distribution Function (CCDF) of the PAPR obtained by the proposed technique (with $D=2,4,8,16$ ), the TR algorithm defined in DVB-T2 [1] (with a clipping threshold of $7 \mathrm{~dB}$ and 16 iterations), the clipping technique (with clipping level at $75 \%$ of the maximum of the original OFDM symbols) and the optimal PTS (with $S=16$ subblocks and rotation angles $\{0, \pi\}$ ). It can be observed that for any $D$ larger than 2, our proposal achieves a better PAPR reduction than the TR algorithm. Note that, in contrast to the TR technique, our proposal avoids any spectral spoilage. Moreover, the proposed method outperforms the clipping technique for any $D$ larger than 4 . It is worth mentioning 
TABLE I

COMPARISON OF TOTAL COMPUTATIONAL COMPLEXITIES (M1: MAX-LOG, M2: MMSE-BASED DETECTOR, M3: PROPOSAL, $r_{a}>0$, M4: PROPOSAL, $r_{a}=0$ AND M5: ML, BLIND SLM [26]).

\begin{tabular}{|c|c|c|}
\hline & RS & RC \\
\hline \hline M1 & $D N(3 M+1)-D$ & $D(N(M-1)+1)-1$ \\
\hline M2 & $15 D N$ & $D N\left(2 \log _{2} M-2\right)$ \\
\hline M3 & $\left(N\left(2+r_{a}(2+8 D)+D\right)-D\right.$ & $\left(4+D\left(4 r_{a}-1\right)\right) N+D-1$ \\
\hline M3 & $N(3+5 D)-D$ & $(N+1) D-1$ \\
\hline M5 & $D N(3+3 M)$ & $D-1$ \\
\hline
\end{tabular}

\begin{tabular}{|c|c|c|}
\hline & RM & RI \\
\hline \hline M1 & $4 N M D$ & 0 \\
\hline M2 & $38 N D$ & $D N$ \\
\hline M3 & $N\left(1+r_{a}(4+8 D)\right)$ & $2 N$ \\
\hline M4 & $N(3+4 D)$ & $2 N$ \\
\hline M5 & $D(N(4+2 M))+1$ & 0 \\
\hline
\end{tabular}

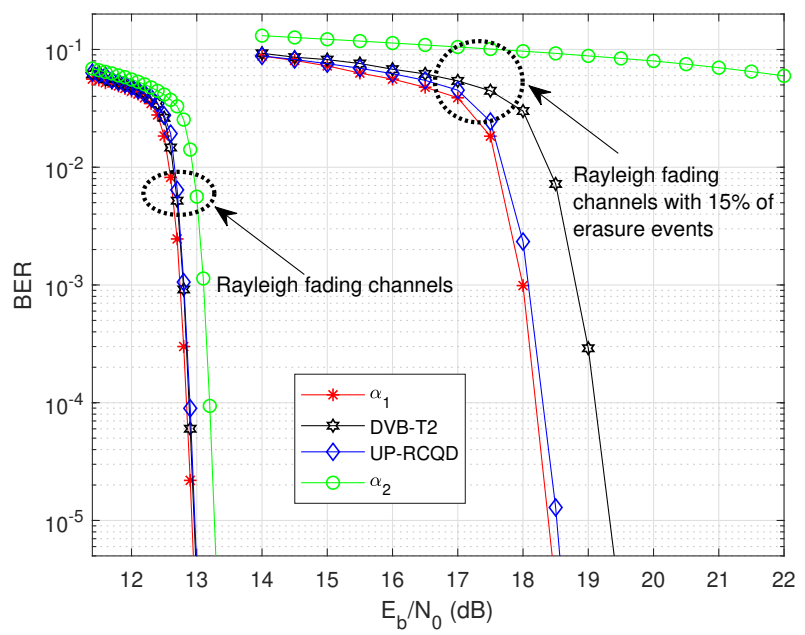

Fig. 5. BER comparison for the 64-QAM over Rayleigh fading channels with and without erasure.

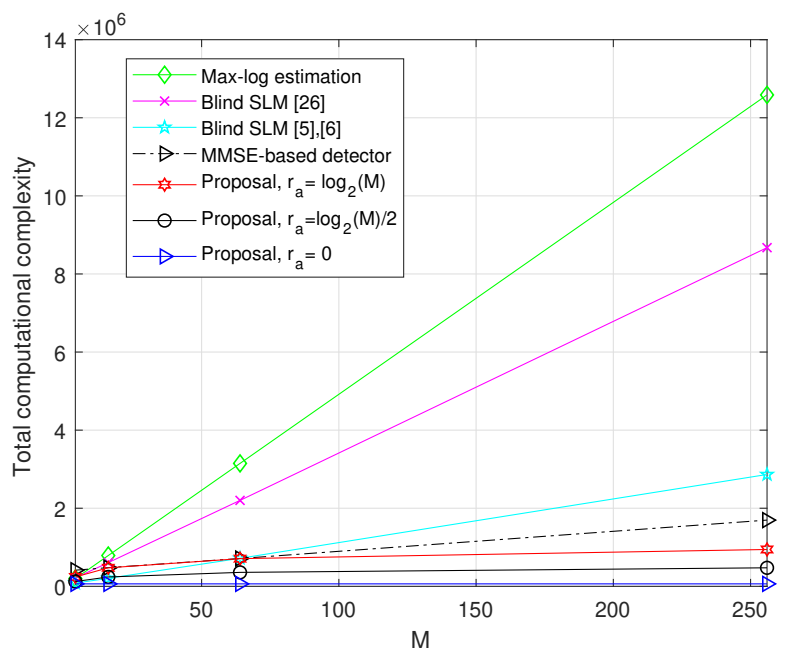

Fig. 6. Computational complexity comparison for $N=256$ and $D=16$.

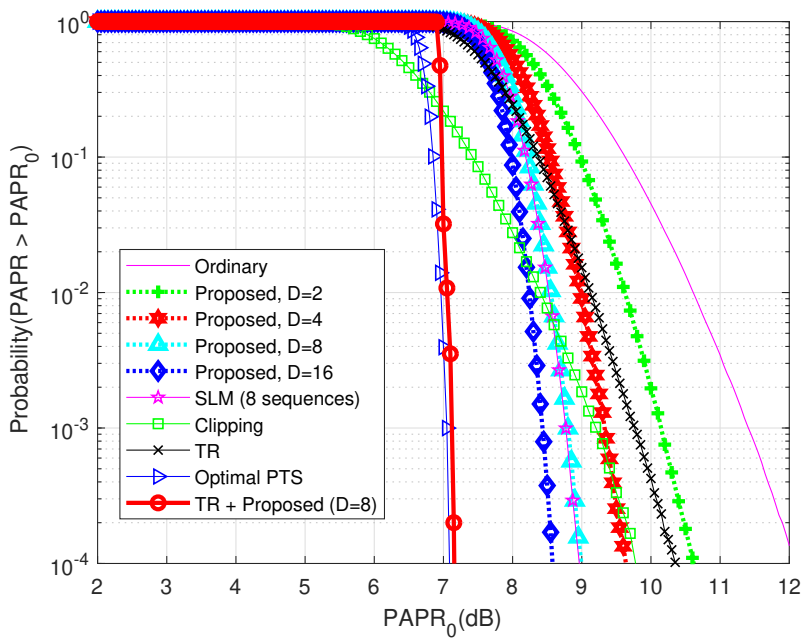

Fig. 7. CCDFs of the PAPR for the 64-QAM constellation and $N=1024$.

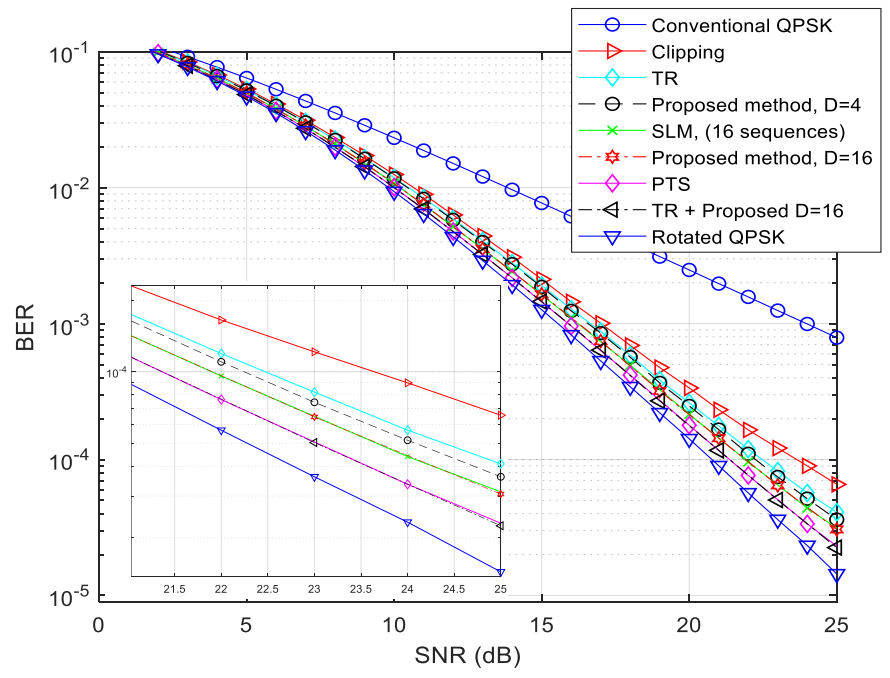

Fig. 8. BER comparison between several PAPR reduction schemes.

that the clipping technique may lead to a serious BER degradation, especially for low clipping thresholds [11]. Furthermore, the optimal PTS allows a good PAPR reduction performance; however, this gain is obtained at the expense of a high exponential complexity in $\mathrm{S}$ and a large side information; therefore, several low-complexity PTS schemes have been proposed but they may lead to suboptimal PAPR reduction performance [19]. Besides, when we jointly use the TR and the proposed algorithms with $D=8$, we obtain similar PAPR reduction performance to the optimal PTS with much lower computational complexity and higher spectral efficiency. Finally, when compared to the blind SLM method [5], [6], both methods have approximately the same PAPR reduction performance; however, our proposal for UPRCQD constellations reduces considerably the computational complexity both at the transmitter and the receiver sides; in particular, in addition to the computational complexity 


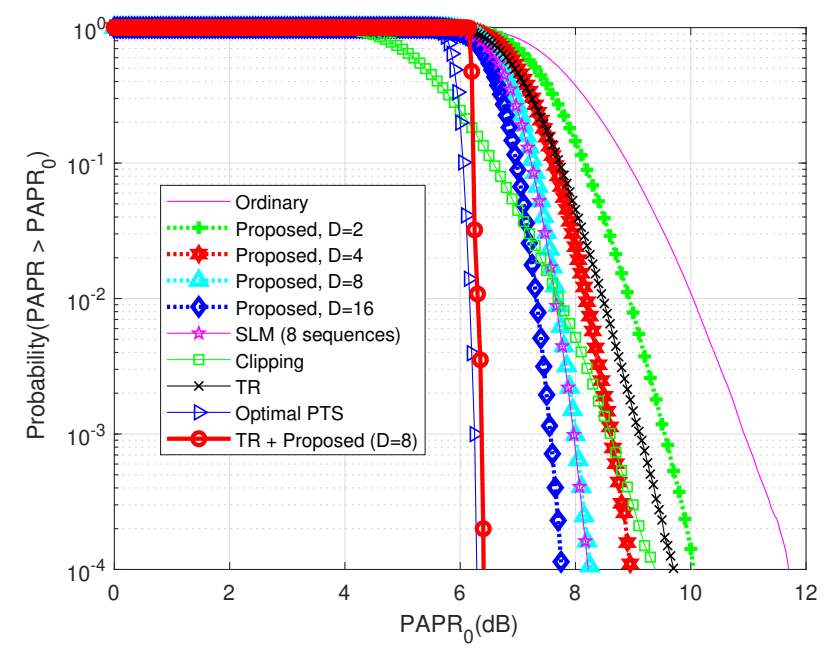

Fig. 9. CCDFs of the PAPR for the 16-QAM constellation and $N=256$.

reduction obtained for the LLR computation thanks to the use of the UP-RCQD constellation [9], the computational complexity is reduced, with the chosen system parameters, by $97 \%$ (see Table I and Fig. 6).

Fig. 8 depicts the BER performance versus SNR of OFDM signals with several PAPR reduction schemes over the Rayleigh fading channel, in which the typical HPA of the Solid State Power Amplifier (SSPA) has been considered [11], [27]:

$$
\left|v_{\text {out }}\right|=\frac{\left|v_{\text {in }}\right|}{\left(1+\left(\frac{\left|v_{\text {in }}\right|}{v_{\text {sat }}}\right)^{2 p}\right)^{\frac{1}{2 p}}},
$$

where $v_{\text {in }}$ is the complex input, $v_{\text {out }}$ is the complex output and $v_{\text {sat }}$ is the output saturation level. In our simulations, $v_{\text {sat }}$ (resp. $p$ ) is set to 1 (resp. 4), the amplifier input back-off is set to $3 \mathrm{~dB}$ [27], and the number of subcarrier is equal to 1024. Moreover, in Fig. 8, the performance of the conventional QPSK and the rotated QPSK constellations are obtained by ignoring the effect of the SSPA and directly transmitting the original OFDM signals through the channel.

It can be observed the great impact of Signal Space Diversity on the system performance: the rotated QPSK achieves roughly a $10 \mathrm{~dB}$ gain in performance compared to the conventional QPSK thanks to its inherent diversity. We can also observe that the capability of the PAPR reduction of a given method considerably influences its BER performance. The proposed algorithm with $\mathrm{D}=16$ leads to the best BER improvement.

Furthermore, Fig. 9 confirms the results of Fig. 7 for other system parameters. Note finally that, all the considered methods in Fig. 7 to Fig. 9 do not have the same computational complexity and beyond the capability of the PAPR reduction by itself, when choosing a PAPR reduction method, other criteria need to be considered in order to meet the requirements of the communication system, such as the BER performance, the spectral efficiency and the computational complexity [11].

\section{CONCLUSION}

This paper proposes a new blind interleaving technique for PAPR reduction without any distortion of OFDM systems using signal space diversity. It is based on the use of rotated constellations as they allow the receiver to identify, without any SI, the two-component interleaver used in the broadcast. To lower down the complexity burden at the receiver side, an MMSE-based detector with good estimation performance is proposed. Furthermore, in order to reduce even more the computation complexity of the demapper, we propose to use the UP-RCQD constellation as this constellation brings many interesting structural properties that we employ to design a low-complexity interleaver index decoder. The main advantages of this technique are as following. Our proposal achieves a large PAPR reduction performance without neither BER degradation, nor any spectral spoilage. In addition, compared to the blind SLM method, the proposed technique simplifies both the transmitter and the receiver design and its lowcomplexity makes it particularly suited for hardware implementation.

\section{REFERENCES}

[1] Implementation guidelines for a second generation digital terrestrial television broadcasting system (DVB-T2), European Telecom. Standard Institute ETSI TR 102831 V1.1.1, Oct. 2010.

[2] J. Boutros, and E. Viterbo, "Signal space diversity: a power- and bandwidth-efficient diversity technique for the Rayleigh fading channel", IEEE Trans. on Inform. Theory, 44 (4), pp. 1453-1467, July 1998.

[3] J. Kim, I. Lee, "Analysis of symbol error rates for signal space diversity in Rayleigh fading channels", IEEE Int. Conf. Commun. (ICC), pp. 4621-4625, 2008.

[4] Q. Xie, J. Song, K. Peng, and F. Yang, "Coded modulation with signal space diversity," IEEE Trans. on Wireless Communication, 10 (2), pp. 660-669, Feb. 2011

[5] T. Arbi and B. Geller, "Joint BER Optimization and Blind PAPR Reduction of OFDM Systems with Signal Space Diversity," IEEE Communications Letters, 23 (10), pp. 1866-1870, Oct. 2019.

[6] T. Arbi, I. Nasr and B. Geller, "Near Capacity RCQD Constellations for PAPR Reduction of OFDM Systems,'IEEE Inter. Conf. on Acoustics, Speech and Signal Processing (ICASSP), Barcelona, Spain, pp. 51105114, May 2020.

[7] K. Kim, K. Bae, and H. Yang, "One-dimensional soft-demapping using decorrelation with interference cancellation for rotated QAM constellation," Proc. 2012 IEEE Conf. Comm. and Net. Conference (CCNC), pp. 787-791, Jan. 2012.

[8] S. Tomasin, and M. Butussi, "Low complexity demapping of rotated and cyclic Q delayed constellation for DVB-T2," IEEE Wireless Comm. Letters, 1 (2), pp. 81-84, April 2012.

[9] T. Arbi, B. Geller, J. Yang, C. Abdel Nour and O. Rioul, "Uniformly Projected RCQD QAM: A Low-Complexity Signal Space Diversity Solution Over Fading Channels With or Without Erasures," IEEE Trans. on Broadcasting, 64 (4), pp. 803-815, Dec. 2018.

[10] A. R. Jafri, A. Baghdadi, M. Waqas, and N. Ul-Islam, "High-Throughput and Area-Efficient Rotated and Cyclic Q Delayed Constellations Demapper for Future Wireless Standards," IEEE Access , 5 (1), pp. 3077-3084, Jan. 2017.

[11] T. Jiang and Y. Wu, "An Overview: Peak-to-Average Power Ratio Reduction Techniques for OFDM Signals," IEEE Trans. on Broadcasting, 54 (2), pp. 257-268, June 2008.

[12] Y. Wang and Z. Luo, "Optimized Iterative Clipping and Filtering for PAPR Reduction of OFDM Signals," IEEE Trans. on Communications, 59 (1), pp. 33-37, Jan. 2011. 
[13] P. Yu and S. Jin, "A Low Complexity Tone Reservation Scheme Based on Time-Domain Kernel Matrix for PAPR Reduction in OFDM Systems," IEEE Trans. on Broadcasting, 61 (4), pp. 710-716, Dec. 2015.

[14] S. S. K. C. Bulusu, M. Crussière, J. F. Hélard, R. Mounzer, Y. Nasser, O. Rousset, and A. Untersee, "Quasi-Optimal Tone Reservation PAPR Reduction Algorithm for Next Generation Broadcasting Systems: A Performance/Complexity/Latency Tradeoff With Testbed Implementation," IEEE Trans. on Broadcasting, 64 (4), pp. 883-899, Dec. 2018.

[15] A. Ghassemi and T. A. Gulliver, "Partial Selective Mapping OFDM with Low Complexity IFFTs," IEEE Communications Letters, 12 (1), pp. 4-6, Jan. 2008.

[16] D. J. G. Mestdagh, J. L. Gulfo Monsalve and J.-M. Brossier, "GreenOFDM: a new selected mapping method for OFDM PAPR reduction," IEE Electronics letters, vol. 54 (7), pp. 449-450, March 2018

[17] D. J. G. Monsalve, D. J. G. Mestdagh and J.-M. Brossier, "Computational complexity reduction of GreenOFDM," Ann. Telecommun. 75, 185-191, DOI: 10.1007/s12243-019-00738-2, Jan. 2020.

[18] A. D. S. Jayalath and C. Tellambura, "The use of interleaving to reduce the peak-to-average power ratio of an OFDM signal," IEEE. Global Telecommunications Conference Globecom, San Francisco, USA, pp. 82-86, Aug. 2000.

[19] Y. A. Jawhar, L. Audah, M. A. Taher, K. N. Ramli, N. S. M. Shah, M. Musa, and M. S. Ahmed, "A Review of Partial Transmit Sequence for PAPR Reduction in the OFDM Systems," IEEE Access, 7, pp. 18021$18041,2019$.

[20] K. Lee, H. Kang and J. No, ”New PTS Schemes With Adaptive Selection Methods of Dominant Time-Domain Samples in OFDM Systems," IEEE
Trans. on Broadcasting, 64 (3), pp. 747-761, Sept. 2018.

[21] H. Joo, S. Heo, H. Jeon, J. No and D. Shin, "A New Blind SLM Scheme With Low Decoding Complexity for OFDM Systems," IEEE Trans. on Broadcasting, 58 (4), pp. 669-676, Dec. 2012.

[22] K. Bae, J. G. Andrews and E. J. Powers, "Adaptive active constellation extension algorithm for peak-to-average ratio reduction in OFDM," IEEE Communications Letters, 14 (1), pp. 39-41, Jan. 2010.

[23] H. G. Ryu, S. K. Kim and S. B. Ryu, "Interleaving method without side information for the PAPR reduction of OFDM system," Int. Sympo. on Commun. and Information Technologies, Sydney, pp. 72-76, Oct. 2007.

[24] A. Younes, B. Boubakar, S Bachir and C. Duvanaud, " Interleaving Technique Implementation to Reduce PAPR of OFDM Signal in Presence of Nonlinear Amplification with Memory Effects," Journal of Telecommunications and Information Technology, DOI: 3. 10.26636/jtit.2018.123517, April 2018.

[25] S. Y. Le Goff, S. S. Al-Samahi, B. K. Khoo, C. C. Tsimenidis and B. S. Sharif, "Selected mapping without side information for PAPR reduction in OFDM," IEEE Trans. on Wireless Communications, 8 (7), pp. 33203325 , July 2009.

[26] A. Boonkajay and F. Adachi, "2-Step phase rotation estimation for Low-PAPR signal transmission using blind selected mapping," IEEE 28th Annual Inter. Sym. on Personal, Indoor, and Mobile Radio Comm. (PIMRC), Montreal, pp. 1-5, Oct. 2017.

[27] T. Jiang and G. X. Zhu, "Nonlinearcompandingtransformforreducing peak-to-average power ratio of OFDM signals," IEEE Trans. Broadcasting, 50 (3), pp. 342-346, Sept. 2004. 